\title{
STRONG CONVERGENCE OF RESOLVENTS OF MONOTONE OPERATORS IN BANACH SPACES
}

\author{
KAZUO KIDO
}

(Communicated by John B. Conway)

\begin{abstract}
A BSTRACT. Let $E^{*}$ be a real strictly convex dual Banach space with a Fréchet differentiable norm, and $A$ a maximal monotone operator from $E$ into $E^{*}$ such that $A^{-1} 0 \neq \varnothing$. Fix $x \in E$. Then $J_{\lambda} x$ converges strongly to $P x$ as $\lambda \rightarrow \infty$, where $J_{\lambda}$ is the resolvent of $A$, and $P$ is the nearest point mapping from $E$ onto $A^{-1} 0$.
\end{abstract}

1. Introduction. Let $E$ be a real Banach space, $I$ the identity, and $J$ the (normalized) duality mapping from $E$ into $E^{*}$. Let $B$ be an $m$-accretive operator in $E$ such that $B^{-1} 0 \neq \varnothing$. Then Reich [10] proved that, for every $x \in E, J_{\lambda} x=$ $(I+\lambda B)^{-1} x$ converges strongly to $Q x$ as $\lambda \rightarrow \infty$ when $E$ is uniformly smooth, where $Q$ is the unique sunny and nonexpansive retraction from $E$ onto $B^{-1} 0$. This theorem is useful to obtain strong convergence results for several explicit and implicit iteration methods for accretive operators, see [10].

The purpose of this paper is to obtain the analogous result for a maximal monotone operator $A$ from $E$ into $E^{*}$, which will be crucial to study iterations for monotone operators in Banach spaces. Suppose that $A^{-1} 0 \neq \varnothing$. We know that, for every $z \in E^{*},(J+\lambda A)^{-1} z$ converges strongly to $R z$ as $\lambda \rightarrow \infty$ when $E^{*}$ is strictly convex and has a Fréchet differentiable norm, where $R z$ is the unique element of $A^{-1} 0$ satisfying

$$
\langle z-J(R z), R z-y\rangle \geq 0 \quad \text { for every } y \in A^{-1} 0,
$$

see Reich [9] and also [3, 4]. In this paper we study another convergence theorem to an element of $A^{-1} 0$. Under some conditions, resolvents $J_{\lambda}: E \rightarrow E, \lambda>0$, are defined for $A$, see $\S 2$. Then we prove that, for every $x \in E, J_{\lambda} x$ converges strongly to $P x$ as $\lambda \rightarrow \infty$ when $E^{*}$ has a Fréchet differentiable norm, where $P$ is the unique nearest point retraction from $E$ onto $A^{-1} 0$. The contrast of these results becomes more striking when we characterize retractions, $P$ and $Q$, analytically. That is,

$$
Q x \text { satisfies }\langle x-Q x, J(Q x-y)\rangle \geq 0 \text { for all } y \in B^{-1} 0,
$$

and

$P x$ satisfies that for every $y \in A^{-1} 0$, there is $z \in J(x-P x)$ such that $\langle z, P x-y\rangle \geq 0$,

see $[5,7]$, and also $[8]$ for extensive study concerning such retractions.

Finally, let us consider briefly finding a sequence converging to a zero of the maximal monotone operator $A$. Fix an initial value $x$ in $E$. Then, using the above

Received by the editors March 3, 1987.

1980 Mathematics Subject Classification (1985 Revision). Primary 47H05, 47H15.

Key words and phrases. Monotone operator, resolvent, nearest point, iteration. 
result, $J_{\lambda} x$ approximates $P x \in A^{-1} 0$ well for a sufficiently large $\lambda$ (and for varying initial values, we obtain several elements of $A^{-1} 0$ ). On the other hand, under some conditions, we obtain a sequence converging to $J_{\lambda} x$ by a gradient method, see Theorem 2 and Remark 5. Then, this sequence will be a good approximation to $P x$ if $\lambda$ is sufficiently large. It is an open problem to approximate zeros of monotone operators in Banach spaces by doubly iterations.

2. Main results. Let $E^{*}$ be a real strictly convex dual Banach space with a Fréchet differentiable norm, and $J$ be the (normalized) duality mapping from $E$ into $E^{*}$, i.e., $J x=\left\{y \in E^{*}:\langle x, y\rangle=\|x\|^{2}=\|y\|^{2}\right\}$ for $x \in E$. Let $A$ be a (multivalued) maximal monotone operator from $E$ into $E^{*}$, i.e., $\left\langle y_{1}-y_{2}, x_{1}-x_{2}\right\rangle \geq 0$ for all $y_{1} \in A x_{1}, y_{2} \in A x_{2}: x_{1}, x_{2} \in D(A)$, and $A$ has no monotone extension. Fix $x \in E$. Then for every $\lambda>0$ there exists a unique $x_{\lambda} \in D(A)$ such that $0 \in J\left(x_{\lambda}-x\right)+\lambda A x_{\lambda}$ (see [1, p. 104]). Putting $J_{\lambda} x=x_{\lambda}$, we define the resolvent $J_{\lambda}: E \rightarrow E$ of $A$ for every $\lambda>0$. Next, since $A$ is a maximal monotone, $A^{-1} 0$ is closed convex. If $A^{-1} 0 \neq \varnothing$ then the strict convexity of $E$ ensures the unique existence of the nearest point retraction $P$ of $E$ onto $A^{-1} 0$. Then we prove

THEOREM 1. Let $E^{*}$ be a real strictly convex dual Banach space with a Fréchet differentiable norm, and $A$ a maximal monotone operator from $E$ into $E^{*}$ such that $A^{-1} 0 \neq \varnothing$. Then, for every $x \in E, J_{\lambda} x$ converges strongly to $P x$ as $\lambda \rightarrow \infty$.

PROOF. Fix $\lambda>0$ arbitrarily. Then from the definition of $x_{\lambda}\left(=J_{\lambda} x\right)$ there exists $y_{\lambda} \in E^{*}$ such that $y_{\lambda}$ belongs to both $J\left(x-x_{\lambda}\right)$ and $\lambda A x_{\lambda}$. For every $v \in A^{-1} 0$, since $A$ is monotone, we have

$$
0 \leq\left\langle y_{\lambda}, x_{\lambda}-v\right\rangle=\left\langle y_{\lambda},\left(x_{\lambda}-x\right)+(x-v)\right\rangle \leq-\left\|x_{\lambda}-x\right\|^{2}+\left\|x_{\lambda}-x\right\| \cdot\|x-v\| .
$$

Therefore we obtain

$$
\left\|x_{\lambda}-x\right\| \leq\|x-v\| \text { for all } v \in A^{-1} 0 \text { and } \lambda>0 .
$$

Next, we show the weak convergence of $x_{\lambda}$ to $P x$. By the inequality (1), we have $\left\|y_{\lambda}\right\| / \lambda=\left\|x-x_{\lambda}\right\| / \lambda \leq\|x-v\| / \lambda \rightarrow 0$ as $\lambda \rightarrow \infty$. Since $E$ is reflexive we can take a subnet $\left\{x_{\lambda_{\alpha}}\right\}$ of $\left\{x_{\lambda}\right\}$ such that $x_{\lambda_{\alpha}}$ converges weakly to some $\bar{x} \in E$. Then since $\left(x_{\lambda_{\alpha}}, y_{\lambda_{\alpha}} / \lambda_{\alpha}\right) \in A$ and $A$ is maximal monotone, $\bar{x} \in A^{-1} 0$. Therefore by using (1) and the weak convergence of $x-x_{\lambda_{\alpha}}$ to $x-\bar{x}$, we have

$$
\|x-\bar{x}\| \leq \liminf _{\alpha}\left\|x-x_{\lambda_{\alpha}}\right\| \leq\|x-v\| \quad \text { for all } v \in A^{-1} 0 .
$$

Thus $\bar{x}=P x$. Since every convergent subnet has a unique convergent element $P x$, $x_{\lambda}$ itself converges weakly to $P x$ as $\lambda \rightarrow \infty$.

Then we obtain, as (2),

$$
\|x-P x\| \leq \liminf _{\lambda}\left\|x-x_{\lambda}\right\| \leq \limsup _{\lambda}\left\|x-x_{\lambda}\right\| \leq\|x-P x\| .
$$

That is $\left\|x-x_{\lambda}\right\|$ converges to $\|x-P x\|$ as $\lambda \rightarrow \infty$. Since $E^{*}$ has a Fréchet differentiable norm, this implies the strong convergence of $x-x_{\lambda}$ to $x-P x$. Equivalently we obtain $x_{\lambda} \rightarrow P x$ as $\lambda \rightarrow \infty$.

REMARK 1 . Instead of the normalized duality mapping $J$, the analogous result holds for the duality mapping $J_{\phi}$ with a gauge function $\phi$.

REMARK 2. Fix $x \in E$. Instead of the exact form of $J_{\lambda} x$, let $x_{\lambda} \in E, \lambda>0$, be a unique element satisfying $\varepsilon_{\lambda} \in J\left(x_{\lambda}-x\right)+\lambda A x_{\lambda}$ in $E^{*}$. If $\varepsilon_{\lambda}$ converges to 0 
as $\lambda \rightarrow \infty$ in $E^{*}$, then the same result as in Theorem 1 follows, i.e., $x_{\lambda} \rightarrow P x$ as $\lambda \rightarrow \infty$.

REMARK 3. From Theorem 1 and the proof of it, we have $A^{-1} 0=\varnothing$ if and only if $\lim _{\lambda \rightarrow \infty}\left\|J_{\lambda} x\right\|=\infty$.

REMARK 4 . In the definition of $J_{\lambda} x$, the strict convexity of $E^{*}$ is needed only to assert the existence of $J_{\lambda} x$ by using Corollary 4.1 of [1]. Therefore it is dropped when $R(J(\cdot-x)+\lambda A(\cdot))=E^{*}$ is claimed by another reason. We say a monotone operator $A$ from $E$ into $E^{*}$ satisfying such a condition is an $m$-monotone operator (with respect to $J$ ). When $E^{*}$ is a strictly convex Banach space with a Fréchet differentiable norm, a maximal monotone operator from $E$ into $E^{*}$ is $m$ monotone. Another example of an $m$-monotone operator is the subdifferential of a lower-semicontinuous, proper and convex function on a reflexive Banach space. Then Theorem 1 holds if $E^{*}$ has a Fréchet differentiable norm, and if $A$ is a (multivalued) $m$-monotone operator from $E$ into $E^{*}$ such that $A^{-1} 0 \neq \varnothing$.

Finally, we show a theorem to obtain the resolvent.

THEOREM 2. Let $E^{*}$ be a real dual Banach space with a Fréchet differentiable norm, $J$ the (normalized) duality mapping from $E$ into $E^{*}$, and $A$ an $m$-monotone operator from $E$ into $E^{*}$. Fix $x \in E$ and $\lambda>0$. Define a monotone operator $B$ from $E$ into $E^{*}$ by $B(y)=J(y-x)+\lambda A(y), y \in D(A)$. Then if $\left\{\left(x_{n}, y_{n}\right)\right\}$ is a sequence in the graph of $B$ such that $\left\{x_{n}\right\}$ is bounded and $y_{n} \rightarrow 0$ as $n \rightarrow \infty$, then $x_{n}$ converges strongly to $J_{\lambda} x$ as $n \rightarrow \infty$.

PROOF. Let $y_{n}=p_{n}+q_{n}, p_{n} \in J\left(x_{n}-x\right), q_{n} \in \lambda A x_{n}$, and $r \in J\left(J_{\lambda} x-x\right) \cap$ $-\lambda A\left(J_{\lambda} x\right)$. Then we obtain

$$
\begin{aligned}
\left\langle y_{n}, x_{n}-J_{\lambda} x\right\rangle & =\left\langle p_{n}+q_{n}, x_{n}-J_{\lambda} x\right\rangle \\
& =\left\langle p_{n}-r, x_{n}-J_{\lambda} x\right\rangle+\left\langle q_{n}+r, x_{n}-J_{\lambda} x\right\rangle \\
& \geq\left\langle p_{n}-r, x_{n}-J_{\lambda} x\right\rangle .
\end{aligned}
$$

Since $\left\{x_{n}\right\}$ is bounded and $y_{n}$ converges strongly to 0 , the left-hand side of the above inequality tends to 0 as $n \rightarrow \infty$. Therefore $\lim _{n}\left\langle p_{n}-r,\left(x_{n}-x\right)-\left(J_{\lambda} x-x\right)\right\rangle=$ 0 . Remark that $p_{n} \in J\left(x_{n}-x\right)$ and $r \in J\left(J_{\lambda} x-x\right)$. Since $E^{*}$ has a Fréchet differentiable norm, this implies that $x_{n}-x$ converges strongly to $J_{\lambda} x-x$ as $n \rightarrow \infty$, equivalently $x_{n}$ converges strongly to $J_{\lambda} x$ as $n \rightarrow \infty$.

REMARK 5 . When $A$ is the subdifferential of a lower-semicontinuous, proper and convex function $f$ on $E$, then $B$ is the subdifferential of $g(y)=\|y-x\|^{2} / 2+$ $\lambda f(y), y \in D(f)$. Then, under some additional assumptions, a sequence $\left\{x_{n}\right\}$ satisfying the whole condition of Theorem 2 is obtained by a gradient method for $g$, see [2].

ACKNOWLEDgement. The author wishes to express his hearty thanks to Professor W. Takahashi for many kind suggestions and advice in the course of preparing the present paper, and also to the referee for his many helpful suggestions.

\section{REFERENCES}

1. V. Barbu and Th. Precupanu, Convexity and optimization in Banach spaces, Editura Academiei R. S. R., Bucuresti, 1978.

2. E. K. Blum, Numerical analysis and computation: theory and practice, Addison-Wesley, 1972. 
3. H. Brezis and M. Sibony, Méthodes d'approximation et d'itération pour les opérateurs monotones, Arch. Rational Mech. Anal. 28 (1968), 59-82.

4. F. E. Browder, Existence and approximation of solutions of nonlinear variational inequalities, Proc. Nat. Acad. Sci. U.S.A. 56 (1966), 1080-1086.

5. R. E. Bruck, Non-expansive retracts of Banach spaces, Bull. Amer. Math. Soc. 76 (1970), 384-386.

6. __ A strongly convergent iteration of $0 \in U(x)$ for a maximal monotone operator $U$ in Hilbert space, J. Math. Anal. Appl. 48 (1974), 114-126.

7. F. R. Deutsch and P. H. Maserick, Application of the Hahn-Banach theorem in approximation theory, SIAM Rev. 9 (1967), 516-530.

8. K. Goebel and S. Reich, Uniform convexity, hyperbolic geometry, and nonexpansive mappings, Marcel Dekker, New York and Basel, 1984.

9. S. Reich, Constructive techniques for accretive and monotone operators, Applied Nonlinear Analysis (V. Lakshmikantham, Ed.), Academic Press, New York, 1979, pp. 335-345.

10. Strong convergence theorems for resolvents of accretive operators in Banach spaces, J. Math. Anal. Appl. 75 (1980), 287-292.

DEPARTMENT OF INFORMATION SCIENCE, TOKYO INSTITUTE OF TECHNOLOGY, TOKYO 152, JAPAN

Current address: Software Laboratory, Nippon Rasen Company Limited, 2-8-6-308 Hanegi, Setagaya-ku, Tokyo 156, Japan 